\section{$\underset{\substack{\text { hommes } \\ \text { \& migrations }}}{ }$}

\section{Hommes \& migrations}

Revue française de référence sur les dynamiques

migratoires

$1297 \mid 2012$

Migrations en création

\title{
Un hymne à l'art par-delà les frontières
}

Paroles d'un plasticien africain devenu créateur international

\section{Jacques Barou}

\section{(2) OpenEdition}

1 Journals

\section{Édition électronique}

URL : http://journals.openedition.org/hommesmigrations/1550

DOI : 10.4000/hommesmigrations. 1550

ISSN : 2262-3353

Éditeur

Musée national de l'histoire de l'immigration

Édition imprimée

Date de publication : 1 mai 2012

Pagination : 88-94

ISSN : 1142-852X

\section{Référence électronique}

Jacques Barou, « Un hymne à l'art par-delà les frontières », Hommes \& migrations [En ligne],

1297 | 2012, mis en ligne le 01 mai 2014, consulté le 21 avril 2019. URL : http://

journals.openedition.org/hommesmigrations/1550; DOI : 10.4000/hommesmigrations. 1550

Tous droits réservés 


\section{Un hymne à l'art par-delà les frontières Paroles d'un plasticien africain devenu créateur international}

Entretien réalisé par Jacques Barou, directeur de recherche au CNRS, UMR PACTE, Grenoble

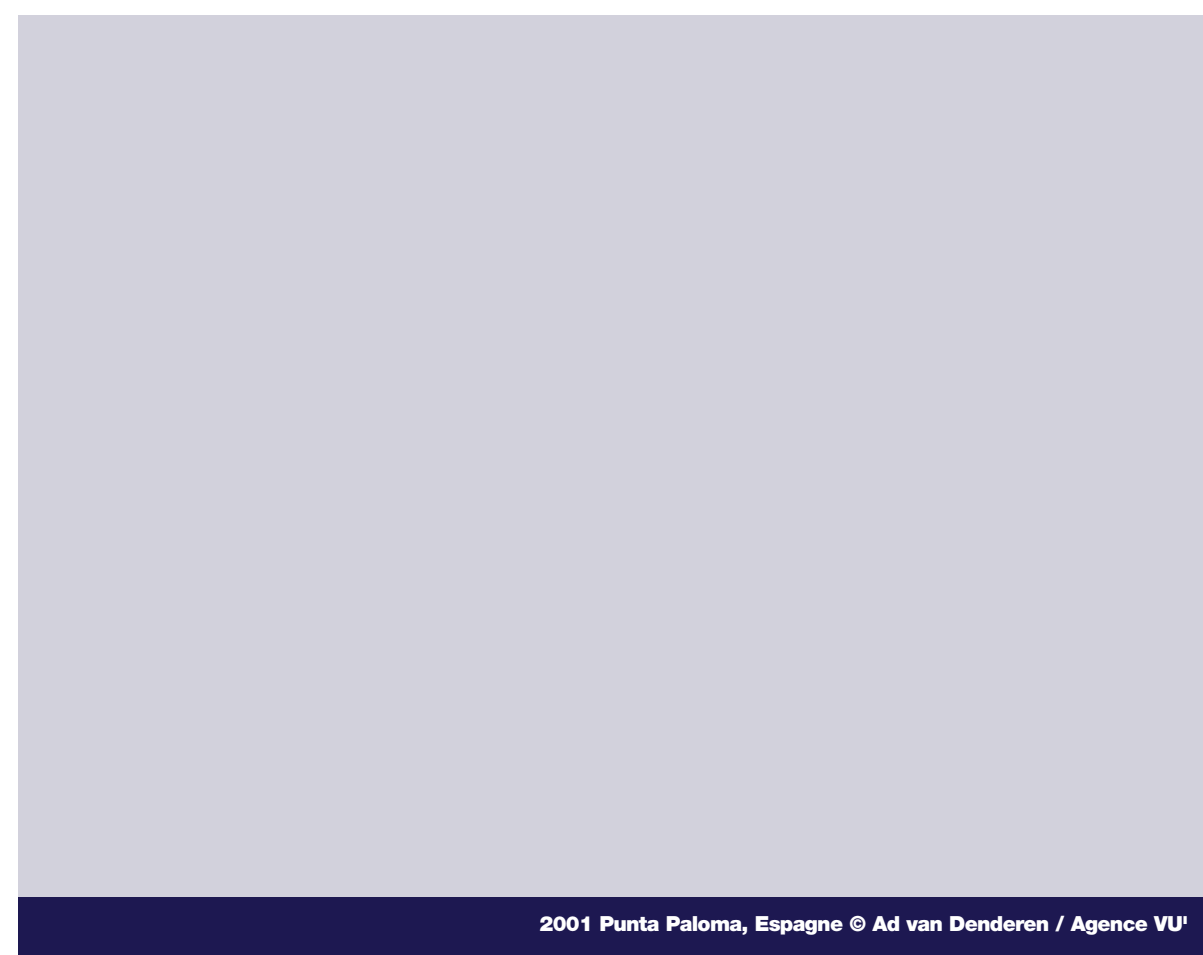

Moridja Kitenge Banza, né en 1980 à Kinshasa et installé en France depuis 2003, est un jeune plasticien qui a obtenu en 2010 à la Biennale de Dakar le prix Léopold Sédar Senghor pour une œuvre vidéo intitulée Hymne à nous. En 2011, il a exposé trois de ses œuvres au Musée dauphinois de Grenoble dans le cadre d'une exposition intitulée :

Ce que nous devons à l'Afrique. 
Dans cet entretien accordé à Hommes \& Migrations, Moridja Kitenge Banza explique comment son expérience de l'immigration, en France d'abord, au Canada ensuite, lui a permis d'enrichir sa vision du monde, de transformer son regard sur l'Afrique et a stimulé son inspiration. L'itinéraire qu'il retrace ici est riche de rencontres et de synthèses d'influences diverses. Il dévoile la construction d'une personnalité artistique qui se situe résolument au confluent des échanges contemporains et aspire avant tout à être reconnue comme telle plutôt que classée dans une catégorie particulière d'artistes.

\section{Hommes \& Migrations : Moridja Kitenge Banza, pouvez-vous tout d'abord vous présenter et récapituler votre itinéraire?}

Moridja Kitenge Banza : Moridja, c'est mon prénom. Mon nom complet est Moridja Kitenge Banza. J'ai été diplômé de l'École des beaux-arts de Kinshasa en 2002. J'ai ensuite enseigné pendant un an à l'École des beaux-arts de Lumumbashi, au Katanga. C'est ma région d'origine. Je suis luba. En 2003, comme la situation politique au pays était tendue, j'ai décidé d'aller continuer mes études ailleurs. J'ai obtenu un visa d'études pour la France et une bourse de mon pays que je n'ai jamais touchée. Je me suis retrouvé à l'École des beaux-arts de Nantes où j'avais été admis. Mes parents avaient quelques connaissances à Nantes et pensaient que je m'y sentirais moins seul. Je n'ai pas pu obtenir d'équivalence de mon diplôme de Kinshasa et j'ai dû reprendre un cursus à zéro. J'ai fait cinq ans d'études à Nantes. J'ai fini en 2008 et comme, par ailleurs, je suis passionné par le social et la politique, j'ai fait un master à l'université de La Rochelle intitulé "Développement culturel des villes". J'ai toujours travaillé en parallèle et cela a nourri mon travail d'artiste. Je ne peux pas créer sans questionner le monde dans lequel je vis.

\section{H\&M : Vous êtes un Africain qui vit depuis plusieurs années en dehors de son pays natal, quelle influence peut avoir votre condition d'immigré sur votre création artistique ?}

Moridja Kitenge Banza : Cette condition a eu deux types de conséquences. Si je reste seul dans mon coin en tant qu'artiste, je ne peux pas questionner le monde qui m'entoure. En tant qu'immigré, on se regarde différemment, on prend conscience que les gens surtout vous regardent différemment. Au Congo, je ne me posais pas la question de savoir si j'étais noir ou africain. C'est en Occident que j'ai pris conscience de ma "congolité", de mon africanité. Édouard Glissant a écrit que les minoritaires sont amenés à revendiquer leur identité parce qu'ils sont isolés au milieu des majoritaires. En France, on m'a incité à m'intégrer sans pour autant prendre en compte ma singularité. Au Canada, on m'incite à m'intégrer mais on fait attention à ma singularité et a ce que celle-ci peut apporter de plus à la communauté canadienne. Cela a beaucoup 
inspiré les questions que je me pose sur ma place dans le monde et ces questions ont pesé sur mon travail artistique.

La deuxième conséquence, c'est que cette condition d'immigré m'a incité à voir mon pays de façon différente, sur le plan politique en particulier. Là-bas, on vous incite à penser que tous les malheurs de l'Afrique viennent de l'Occident. Quand on est immigré, on pèse la part de l'Afrique dans son propre malheur, ce qui ne veut pas dire que l'Occident n'y est pour rien. Dans le cas du Congo,j'estime que le pays lui-même a une part de responsabilité de 70 \% dans ce qui lui est arrivé, les $30 \%$ restants incombant à l'Occident. Ce changement de regard, je veux aussi l'exprimer dans mes créations.

H\&M : Vous avez accepté de participer à l'exposition Ce que l'lsère doit à I'Afrique qui a été organisée en 2010 au Musée dauphinois de Grenoble. Ce musée n'est pas un musée des beaux-arts mais un musée de société fondé et animé par des ethnologues. Pourquoi et comment s'est faite votre participation à cet événement ?

Moridja Kitenge Banza : La rencontre s'est faite par hasard. Olivier Cogne, un des commissaires de l'exposition, avait lu une interview de moi sur le site d'Africultures. Il m'a contacté et expliqué le sens du projet d'exposition: Ce que nous devons à l'Afrique. Les questions de société m'intéressent beaucoup, en particulier les débats autour de l'histoire et de la mémoire, vues sous un angle politique et social. Je pense qu'à aucun moment on ne doit passer son temps à ressasser le passé. On doit plutôt se demander ce que l'on peut faire pour que ce qui s'est passé ne puisse plus jamais revenir. Dans l'esclavage, ce qui m'intéresse, ce n'est pas ce qu'il était autrefois mais la manière dont il persiste aujourd'hui sous d'autres formes. Je ne suis pas responsable de ce qui s'est passé avant 1848 et je ne peux pas reprocher à un jeune Français ou à un jeune Anglais d'aujourd'hui d'en être responsable. Mais nous sommes tous responsables de ce qui se passe aujourd'hui et pourra se passer demain. 


\section{H\&M : Vous avez exposé trois œuvres au cours de cette exposition, pouvez-vous nous les présenter et nous dire ce qu'elles signifient pour vous ? Pourquoi les avez-vous choisies ?}

Moridja Kitenge Banza : J'ai d'abord réalisé une pièce spécialement pour l'exposition en rapport avec sa thématique : ce que nous devons à l'Afrique. Cette pièce qui s'intitule Sans titre est un compteur-montre qui fait défiler une somme en euros représentant ce que l'on pourrait, si c'était possible, payer à toutes les personnes qui ont souffert de l'esclavage et de la colonisation. Mais chaque fois que ce compteur atteint une somme maximale, il revient automatiquement à zéro et repart pour un autre calcul de la somme à donner. Cela veut dire qu'à la fois, on doit tout à l'Afrique et qu'on ne lui doit rien. On ne peut pas quantifier ce que l'on doit à travers tous les faits historiques. En fin de compte, la seule chose que l'on doive, c'est le respect. Il appartient aux Africains de prendre conscience du passé pour penser le futur. Or, mon expérience de l'Occident me permet de constater que les choses n'ont pas fondamentalement changé.

Certains Africains, inconsciemment, pensent que les Blancs leur sont supérieurs. Ce musée m'a permis d'exprimer ma pensée sur des faits de société à travers mes cuvres. J'ai exposé aussi Hymne à nous, cuvre qui m'a valu le premier prix à la Biennale de

Dakar. C'est une vidéo

d'une minute dix où je suis filmé nu trente fois avec des expressions différentes sur la musique de l'ode à la joie de Beethoven. Mais les paroles sont différentes. J'y ai introduit un fragment de l'hymne national congolais, de l'hymne belge, de la marseillaise et l'extrait d'un discours du roi Léopold II incitant les missionnaires à aller évangéliser le Congo, tout cela au milieu des paroles de Schiller.

Par là, j'ai voulu me présenter, dire tout ce que je suis, nu d'abord pour indiquer que c'est ainsi que j'arrive, sans rien, et aussi pour rappeler que mes ancêtres, les guerriers luba, partaient nus se battre à la guerre. Pourquoi tous ces hymnes ? Parce que je suis congolais bien sûr mais parce que j’ai été éduqué dans un système légué par la 
colonisation belge, parce que j'ai appris les valeurs de la République française et parce que j'ai fait partie d'une chorale où on chantait des textes allemands. Cela veut dire que je suis tout cela à la fois, que je suis moi-même, dans ma nudité authentique et aussi le produit de toutes ces influences extérieures qui m’ont façonné. Pourquoi ce titre Hymne à nous parce qu'un Chinois, un Malais ou n'importe qui d'autre peut aussi se retrouver là-dedans.

H\&M : Vous êtes inspiré par le passé du continent africain dans ce qu'il a pu avoir de plus tragique comme l'épisode de la traite négrière, mais vous vous efforcez de mettre en miroir ce passé avec ce que sont actuellement les relations entre l'Afrique et le monde occidental, n'estce pas là le fruit de votre expérience de l'immigration ?

Moridja Kitenge Banza : Cela s'exprime à travers la troisième ceuvre que j’ai exposée et qui s'intitule : De 1848 à nos jours. Je m'inspire d'un fait historique qui est la traite négrière mais c'est pour faire le lien avec les injustices d'aujourd'hui. Pour l'instant, elle se compose de 610 cuillères à café rangées étroitement les unes à côté des autres pour évoquer les esclaves entassés dans la soute d'un bateau négrier. J'achète ces petites cuillères dans différents lieux géographiques, comme les négriers achetaient des esclaves dans différents comptoirs en les évaluant et en donnant leur prix. Chaque fois, c'est moi qui donne le prix des cuillères. Chaque cuillère que j'achète représente symboliquement un esclave qui est racheté et la faute allant avec est aussi rachetée par la même occasion. Ici, en Occident, on parle maintenant de l'esclavage et des responsabilités que l'on y a, alors que je n'ai jamais entendu aucun responsable politique africain reconnaître les responsabilités de l'Afrique dans ce drame. Mon objectif est de parler de l'esclavage pour que ce phénomène ne revienne plus. Je veux faire un lien avec le présent. C'est moi qui donne le prix des petites cuillères,

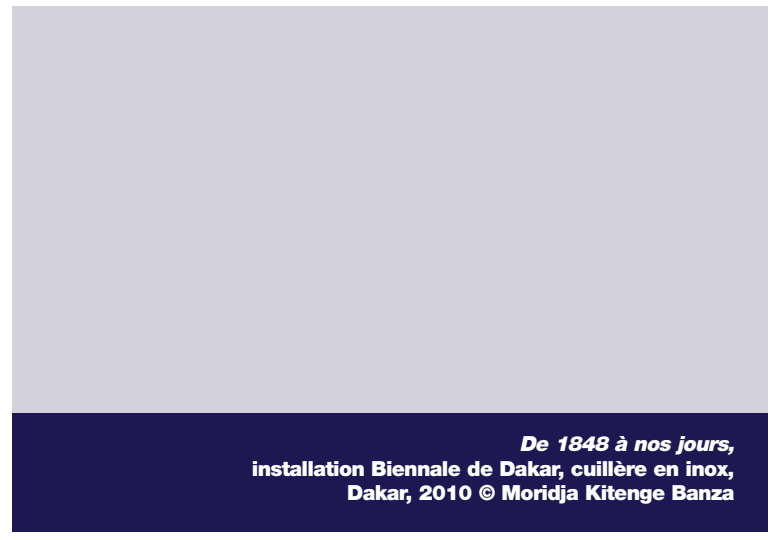
comme ce sont les entreprises occidentales qui donnent le prix des matières premières qu'elles achètent aux pays africains, profitant ainsi d'un rapport de force qui leur demeure favorable. Je ne veux pas rester dans le passé mais je veux le lier au présent. 
H\&M : Le fait d’avoir vécu en France et de vivre actuellement au Canada vous a-t-il apporté des sources d'inspiration que vous n'auriez pas eues si vous étiez resté en Afrique ? Votre expérience de la migration a-t-elle changé votre regard sur l'Afrique?

Moridja Kitenge Banza : Le fait de vivre aujourd'hui au Canada a simplement élargi mon horizon. Quand j'étais au Congo, je rêvais d'une Afrique future mais je la voyais avec des yeux d'Africain. Je rêve maintenant d'une Afrique future évoluant au rythme du monde qui l'entoure. Le fait de vivre en Occident me permet d'imaginer une évolution du Congo en synergie avec tous les autres pays de la planète.

H\&M : Vous trouvez-vous des points communs avec d'autres créateurs immigrés ?

Moridja Kitenge Banza : Oui, la plupart des artistes africains qui ont émigré traitent de sujets politiques et identitaires. C'est une chose que je vois moins chez les artistes occidentaux. On est aussi différents des artistes restés en Afrique. On traite parfois des mêmes sujets mais de façon différente. On a la chance d'avoir un regard que ces derniers n'ont pas.

H\&M : Vous avez commencé vos études et votre carrière de créateur en République Chiromancie \#4, $n^{\circ} 11$, ecoline sur papier polyester; Dakar, 2011 ๑ Moridja Kitenge Banza démocratique du Congo, quel regard portez-vous sur l’art africain et son évolution récente ? Comment intègre-t-il les influences extérieures et comment reflète-t-il ou non les transformations vécues sur le continent ?

Moridja Kitenge Banza : Je ne connais pas tout de l'art africain mais ce que j'en connais me fait dire que cet art a toujours reflété les questions politiques et de société. 
L'Occident n'a pas vu ça, il a voulu voir avant tout des objets exotiques détachés de leur contexte. Il n'y a pas un marché de l'art en Afrique. La grande majorité des acheteurs sont occidentaux. Ce qui fait évoluer l'art africain contemporain, ce sont les biennales. Cela nous permet de nous rencontrer entre artistes africains et de découvrir des sociétés que nous connaissons mal. Il y a un manque d'historiens africains de l'art africain, des gens qui pourraient parler de manière africaine de l'art africain. On a une vision de l'art que l'occidental n'a pas. Des historiens africains pourraient expliquer autrement l'art africain aux Occidentaux.

\section{H\&M : Les artistes comme vous qui ont une expérience internationale peuvent-ils contribuer à un renouvellement de la création plastique en Afrique?}

Moridja Kitenge Banza : Le renouvellement de l'art africain ne se fera qu'en Afrique. Mon souci est de savoir comment créer un marché de l'art en Afrique, comment créer des écoles d'art plastique ayant à la fois une vision africaine et une vision occidentale de l'art. Quand je vais au Congo, je vais dans mon ex-école pour dialoguer avec les étudiants et pas pour leur dire ce qu'il faut faire. Je peux les conseiller. Du fait que j'ai vécu en Europe, je connais les principaux lieux du marché de l'art. Il y a des codes à connaître. Je leur explique quels sont les codes pour présenter leur travail et le rendre visible. Tout ça, ils ne le connaissent pas.

\section{H\&M : Comment vous définiriez-vous : artiste africain, artiste internatio- nal, artiste immigré, artiste tout court ou autrement encore ?}

Moridja Kitenge Banza : Artiste tout court ! Quand on parle d'un Européen, on ne dit pas un artiste européen. Quand on parle d'un Américain, on dit un artiste qui vient des États-Unis. Ce que je veux faire, ce sont des ceuvres où un Chinois ou un Européen puissent se reconnaître aussi bien qu'un Africain. Il est vrai que, jusque-là, j'ai toujours été invité à exposer mes ceuvres dans des expositions qui parlaient de l'Afrique. Je ne le refuse pas bien sûr mais quand est-ce qu'on va considérer qu'un artiste africain est un artiste à part entière ? Une seule fois, en Allemagne, j'ai été invité à exposer non pas parce que j'étais africain mais parce que mes ceuvres rejoignaient la thématique de l'exposition. Je dis toujours que je suis Moridja, artiste. Je suis jeune d'abord, je viens du Congo et je suis africain en dernier lieu. 